\title{
Partner Phubbing, Satisfação e Bem-Estar Subjetivo: O Impacto da Tecnologia Digital nos Relacionamentos
}

\author{
Partner Phubbing, Satisfacción y Bienestar Subjetivo: \\ El Impacto de la Tecnologia Digital en las Relaciones \\ Amorosas
}

\section{(1) Psicogente ISSN 0124-0137 EISSN 2027-212X}

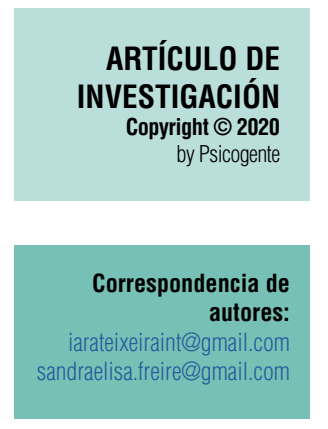

Recibido: 23-11-2019 Aceptado: $31-03-2020$ Publicado: 05-06-2020

\section{Partner phubbing, satisfaction, and subjective well-being: The impact of digital technology on romantic relationships}

\author{
lara do Nascimento Teixeira (iD - Sandra Elisa de Assis Freire (iD \\ Universidade Federal do Piauí, Parnaíba, Brasil
}

Resumo

Objetivo: Este estudo teve por objetivo investigar a influência do Partner Phubbing (Pphubbing) no Bem-Estar subjetivo (BES) e na satisfação com o relacionamento.

Método: Participaram 217 pessoas com média de idade de 25 anos (min. 18, máx. 53 e dp=5,98), sendo $62,2 \%$ do sexo feminino. Estes responderam às escalas de Partner phubbing (Pphubbing), afetos positivos e negativos, satisfação com a vida, satisfação com o relacionamento e questionário sociodemográfico. Os dados foram analisados através do SPSS (v.22).

Resultados: Foi encontrado uma correlação negativa entre Pphubbing e afetos positivos ( $r=-0,32$; $p<0,01)$, satisfação com a vida $(r=-0,13 ; p<0,01)$ e satisfação com o relacionamento $(r=-0,38 p<0,01)$, como também apresentou uma correlação positiva entre Pphubbing e afetos negativos $(r=0,33 ; p<0,01)$. Através da regressão, o Pphubbing mostrou influência na satisfação com o relacionamento ( $\beta=-0,38$; $p<0,001)$, afetos positivos $(\beta=-0,32 ; p<0,001)$ e afetos negativos $(\beta=0,33 ; p<0,001)$.

Conclusões: Os resultados sugerem que os participantes que sofrem mais phubbing de seus parceiros tendem a ter menos satisfação com o relacionamento, assim como menos Bem-Estar Subjetivo. Assim, este é um artigo com resultados inéditos para o Brasil e um dos poucos no mundo a avaliar quantitativamente o phubbing nos relacionamentos amorosos. Desta forma, contribui para o fomento de futuras pesquisas na área e para o embasamento de intervenções e ações de conscientização acerca do uso saudável do Smartphone.

Palavras-Chave: partner phubbing; bem-estar; satisfação com o relacionamento; emoções negativas; emoções positivas.

Resumen

Objetivo: Este estudio tuvo por objetivo investigar la influencia del Partner Phubbing (Pphubbing) en el bienestar subjetivo y la satisfacción en las relaciones amorosas.

Método: En el estudio participaron 217 personas con una media de edad de 25 años (min. 18, máx. 53 y $\mathrm{dp}=5,98$ ), siendo el $62,2 \%$ del sexo femenino. Estos respondieron a las escalas de Pphubbing, afectos positivos y negativos, satisfacción con la vida, escala de satisfacción con la relación y cuestionario sociodemográfico. Los datos fueron analizados a través del SPSS (v.22).

Resultados: Se encontró una correlación negativa y significativa entre el phubbing y los afectos positivos $(r=-0,32, p<0,01)$, la satisfacción con la vida $(r=-0,13 ; p<0,01)$ y la satisfacción con la relación $(r=-0,38$ $p<0,01)$, como también presentó una correlación significativa, positiva y moderada entre el phubbing y

Cómo citar este artículo (APA):

Do Nascimento Teixeira, I. \& De Assis Freire, S. E. (2020). Partner Phubbing, Satisfação e Bem-Estar Subjetivo: O Impacto da Tecnologia Digital nos Relacionamentos. Psicogente 23(44), 1-15. https://doi.org/10.17081/psico.23.44.3438 
los afectos negativos $(r=0,33 ; p<0,01)$. A través de la regresión, el Pphubbing mostró influencia en la satisfacción con la relación $(\beta=-0,38 ; p<0,001)$, afectos positivos $(\beta=-0,32 ; p<0,001)$ y afectos negativos $(\beta=0,33$; $p<0,001)$.

Conclusiones: Los resultados sugieren que los participantes que sufren más phubbing de sus parejas tienden a tener menos satisfacción con la relación, así como menos bienestar subjetivo. Por lo tanto, este es un artículo con resultados inéditos para Brasil y uno de los pocos en el mundo en evaluar cuantitativamente el phubbing en las relaciones amorosas. De esta forma, contribuye al fomento de futuras investigaciones en el área y para el basamento de intervenciones y acciones de concientización acerca del uso saludable del Smartphone.

Palabras clave: partner phubbing; bienestar; satisfacción de la relación; emoción negativa; emoción positiva.

Abstract

Objective: The objective of this study was to research partner phubbing's influence (phubbing) on subjective well-being and romantic relationships' satisfaction.

Method: The study included 217 participants with a mean age of 25 years (minimum age $=18$, maximum age 53 , and $d p=5.98), 62,2 \%$ being female. They responded to the phubbing scales, positive and negative effects, life satisfaction, relationship satisfaction scale, and sociodemographic questionnaire. The data were analyzed through the SPSS software (v.22).

Results: A significant and negative correlation was found between phubbing and positive effects $(r=-0.32$, $p<0.01$ ), life satisfaction $(r=-0.13, p<0.01)$, and relationship satisfaction $(r=-0.38, p<0.01)$, as it also presented a significant, positive, and moderate correlation between phubbing and negative effects $(r=$ $0.33, p<0.01)$. Through the regression, phubbing showed an influence on the relationship satisfaction $(\beta=$ $-0.38, p<0.001)$, positive effects $(\beta=-0.32, p<0.001)$, and negative effects $(\beta=0.33, p<0.001)$.

Conclusions: The results suggest that those who experience more phubbing from their partners tend to be less satisfied with their relationship, as well as have less subjective well-being. Therefore, this article offers unprecedented results for Brazil, one of the few in the world to quantitatively evaluate phubbing in romantic relationships. In this way, it contributes to encouraging future research in the area and provides a basis for interventions and awareness actions regarding the healthy use of smartphone.

Keywords: partner phubbing; well-being; relationship satisfaction; negative emotion; positive emotion.

\section{INTRODUCCIÓN}

A tecnologia de comunicação móvel, em especial o smartphone, tem tomado um papel cada vez maior na vida das pessoas. Dados apontam que de 2000 a 2015 houve o aumento de 806 \% em utilizadores da internet a nível global (a cada 100 habitantes) atingindo a cifra de 4,43 bilhões e, grande parte deste aumento, é devido a dispositivos como smartphones, tablets e notebooks (Patrão \& Sampaio, 2016). Conhecidos como gadgets, estes aparelhos permitem o acesso à internet em qualquer hora ou lugar em decorrência de sua evolução que agrega cada vez mais funções, que vão desde trocas de e-mails à videochamadas e transações bancárias; sendo que as funções mais utilizadas são o acesso às redes sociais e jogos (Karadag et al., 2015). Somente no Brasil já existem 230 milhões de smartphones ativos, anualmente são vendidos 48 milhões de aparelhos, ao passo que os utilizadores de internet relatam a preferência pelo smartphone para acesso (Meirelles, 2019; Ofcom, 2015).

Segundo Perasso (2016), vive-se a quarta revolução tecnológica com o surgimento de nanotecnologias, inteligência artificial e impressoras 3D. A tecno- 
logia tem chegado a todas as áreas da vida humana com o potencial de melhorar a qualidade de vida das pessoas, incluindo seus relacionamentos amorosos. Assim como a tecnologia, os relacionamentos têm passado por mudanças no decorrer dos anos e tiveram seus caminhos cruzados desde a internet em estágios iniciais, enquanto ferramenta para buscar parceiros íntimos e se relacionar (Gillmor, 2007). Desde experimentos com os primeiros computadores existentes em Stanford, os sites de relacionamento como o ParPerfeito e Badoo até os atuais aplicativos de geolocalização para smartphones (Tinder, Happn) onde os usuários tem diversas possibilidade de relacionamento na palma de sua mão, a revolução digital proporcionou a possibilidade de relações outrora inimagináveis entre pessoas que não estão presentes fisicamente e podem nunca se encontrar (Féres-Carneiro, \& Ziviane, 2009; Figueiredo, 2016).

Entretanto, toda esta conectividade entre pessoas distantes tem afastado as que estão próximas. Apesar de estarem próximas fisicamente, não estão inteiramente presentes e disponíveis (Turkle, 2011). Este comportamento é descrito por Misra, Cheng, Genevie \& Yuan (2016) como "absent presence" (presença ausente), onde apesar de estar presente o parceiro necessita competir com o aparelho celular de seu parceiro por atenção, minando não só a disposição em estar presente para o outro como também a capacidade de oferecer um tempo juntos de qualidade. Isto se torna extremamente nocivo para os relacionamentos porque a interação interpessoal entre os parceiros é um dos mais importantes preditores para a satisfação do casal (Bradbury, Fincham, \& Beach, 2000; Kim et al., 2016).

Tornou-se comum estar com o outro e fazer múltiplas checagens ao celular, mesmo sem receber notificações ou ligações, como um comportamento compulsivo (Viacana, Francisquetti, de Oliveira Junior, 2016). Os indivíduos desenvolvem medo de perder eventos, conversas, e experiências que acontecem em seus círculos sociais que são mediados pelo smartphone e, assim, estes indivíduos são levados a inserir o celular em sua rotina, estando sempre conectados em um ritual de checagem do aparelho (Deusen, Bolle, Hegner, \& Kommers, 2015; Chotpitayasunondh, \& Douglas, 2016); comprovando isto em números, há dados que mostram que a maioria das pessoas (89 \%) assume ter levado o celular em sua última interação social, 79 \% usaram o celular em conversas face-a-face e 52 \% já enviaram mensagens a outras pessoas enquanto estavam em um encontro romântico (Harrison, Bealing, \& Salley, 2015; Rainie, \& Zickhur, 2015; Lenhart, \& Duggan, 2014). 
A simples presença deste Smartphone nas interações face a face tem sido preditor de conflitos, redutor da proximidade percebida, da qualidade da conversa e da empatia entre as pessoas próximas (Przybylski, \& Weinstein, 2013; Roberts, \& David, 2016). McDaniel \& Coyne (2016) apontam que o conflito causado em torno disto se dá pelas constantes interrupções para dar atenção ao telefone, transmitindo implicitamente a mensagem de que o telefone é mais importante que o parceiro, interferindo na qualidade do relacionamento. Além disto, do comportamento verbal e não verbal aos seus parceiros, como não fazer contato com os olhos ou não responder imediatamente, pode afetar a imagem que o parceiro tem dele (Abeele, Antheunis, \& Schouten, 2016). Este comportamento em discussão é o foco do presente trabalho e é chamado de partner phubbing, que é o comportamento de usar o smartphone/ser distraído pelo smartphone enquanto está em companhia de outra pessoa (Ugur, \& Koc, 2015). A palavra foi criada a partir do processo conhecido, na língua portuguesa, por neologismo por aglutinação que é a composição de uma nova palavra a partir da junção de duas ou mais palavras. Neste caso, as palavras foram: "phone" (telefone) e "snubbing" (esnobar) (Mcquarie, 2017).

Os estudos sobre Partner phubbing ainda estão em estágio inicial no mundo e, sobretudo, no Brasil. O principal estudo da área promoveu a criação da Partner Phubbing Scale (Roberts, \& David, 2016), uma escala estilo likert que avalia o comportamento do parceiro da pessoa que responde, assim como investigou a influencia do partner phubbing na satisfação com o relacionamento do respondente. A pesquisa chegou à conclusão de que a vida se tornou uma completa distração devido ao smartphone, visto que os resultados apontaram o Pphubbing com um efeito negativo na satisfação do relacionamento, funcionando como um gerador de conflitos. A Partner Phubbing Scale teve suas propiedades psicométricas confirmadas em diversos países, a exemplo de Brasil (Teixeira, \& Freire, in press), Portugal (Água, Coelho, Lourenço, Patrão, \& Leal, 2018), na Turquia (Cizmeci, 2017), Porto Rico (González-Rivera, Segura-Abreu, \& Urbistondo-Rodríguez, 2018) e China (Wang, Xie, Wang, Wang, \& Lei, 2017). O estudo chinês, português e o porto riquenho foram além da validação da escala. Para a amostra coletada na China e para Portugal, o partner phubbing está correlacionado à menor satisfação com o relacionamento amoroso e maiores sintomas depressivos (Wang et al., 2017; Água et al., 2018), para o estudo realizado em Porto Rico, o único estudo realizado em solo latinoamericano até o o momento, sofrer phubbing do parceiro esteve correlacionado à síntomas depressivos, ansiedade, estresse e menor bem-estar psicológico, além de confirmarem o 
papel de mediação da satisfação em relação ao phubbing, bem-estar psicológico e saúde mental.

Esta satisfação, evidenciada nos estudos apresentados, diz respeito à dimensão cognitiva do bem-estar subjetivo e pode ser expressa em diferentes níveis: Satisfação com a vida em geral, satisfação com todo um domínio da vida e satisfação com determinados aspectos de um dominio (Lucas, \& Diener, 2015). A satisfação, que é um julgamento cognitivo feito pelo próprio indivíduo sobre sua vida, pode ser um mediador das emoções, aumentando ou diminuindo as emoções positivas e negativas, assim influenciando felicidade (Diener, Suh, \& Oishi, 1998; Cachioni, Delfino, Yassuda, Batistoni, Melo \& Domingues, 2017). As emoções, ou afetos, dizem respeito à segunda dimensão do bem-estar subjetivo: a dimensão afetiva. Os afetos positivos correspondem a um estado de contentamento puro, entusiasmo, alerta. Altos níveis de afeto positivo significam alta energia, concentração, satisfação; baixos níveis de afeto positivo estão ligados à tristeza; os afetos negativos dizem respeito a uma dimensão geral da angústia e insatisfação, englobando diversos estados de humor aversivo, como o ódio, medo, raiva; baixos níveis de afeto negativo trazem calma e tranquilidade (Watson, Clark, \& Tellegen, 1988). É possível dizer que o sujeito possui bem-estar subjetivo quando há presença de afetos positivos, ausência de afetos negativos e boa satisfação com a vida; alta satisfação com a vida parece estar ligada a um funcionamento positivo, enquanto baixa satisfação com a vida parece relacionada com síntomas depressivos (Portella, de Moura Scortegagna, Pichler \& Graeff, 2017). A meta-análise feita por Proulx, Helms \& Buehler (2007) apontou que o bem-estar subjetivo está intimamente ligado à qualidade do relacionamento, visto que os dois se influenciam. Ou seja, bons níveis de bem-estar subjetivo contribuem para um relacionamento de qualidade ao passo que um relacionamento de qualidade proporciona bem-estar subjetivo.

Diante disso, e da percepção de que a desatenção causada pelo smartphone é capaz de prejudicar a qualidade dos relacionamentos e, também, a importância que o bem-estar subjetivo tem como potencializador de qualidade conjugal, esta pesquisa teve por objetivo investigar a influência do Phubbing no bem-estar subjetivo e na satisfação com o relacionamento dos casais, observando como este fenômeno, que tem se naturalizado nas relações, pode afetar a satisfação com a vida e os afetos positivos e negativos já que poucos ainda são os estudos disponíveis relacionados a este tema; se tornando, assim, o primeiro estudo no Brasil a abordar as variáveis, contribuindo para o fomento de pesquisas futuras e embasamento de intervenções e ações de conscientização sobre o uso saudável do Smartphone. 


\section{MÉTODO}

\subsection{Diseño}

Trata-se de um estudo quantitativo, correlacional, de natureza ex post facto (Shaughnessy, Zechmeister e Zechmeister, 2012).

\subsection{Amostra}

Participaram deste estudo 217 pessoas de forma não probabilística (por conveniência), $62,2 \%$ do sexo feminino, tendo média de idade de 25 anos (min. 18, máx. 53 e dp=5,98). Destes, 71,4 \% estavam namorando, seguido de $21,7 \%$ casados, 5,5\% em um noivado, $0,9 \%$ em outros tipos de relacionamento e $0,5 \%$ eram recasados; o tempo médio de permanência no relacionamento foi de 46,8 meses (min. 6 meses, máx. 324 meses e dp=54,3). Quanto à escolaridade, 52,1\% possuíam Ensino Superior Incompleto, 17,1 \% Ensino Superior Completo, 17,1 \% Pós-graduação, 11,5 \% Ensino Médio Completo, 1,8 \% Ensino Médio Incompleto, e 0,5\% Ensino Fundamental Completo. Estas pessoas foram abordadas em seu ambiente de trabalho, nas ruas, em suas residências e na universidade desde que se disponibilizassem para participar da pesquisa. $O$ estudo teve como critérios de inclusão que o participante tivesse mais de 18 anos e estivesse em um relacionamento amoroso (namoro, noivado, casamento, união estável) com um tempo mínimo de 6 meses de relacionamento. Foram excluídos os casos que não se encaixavam nestes critérios.

\subsection{Instrumentos}

\subsubsection{Partner Phubbing (Pphubbing) Scale - Proposta por Roberts e David (2016).}

Escala do tipo likert de 5 pontos ( $1=$ Nunca; $5=0$ tempo todo) validada para o contexto brasileiro por Teixeira e Freire (in press) que se propõe a mensurar o comportamento de Phubbing nos relacionamentos amorosos. A versão conta com 8 itens em uma estrutura unifatorial e tem por objetivo mensurar o comportamento de Phubbing entre parceiros íntimos. A Escala apresenta confiabilidade de 0,88 , com todos os itens possuindo carga fatorial acima de 0,50 .

\subsubsection{Positive and Negative Affect Schedule (PANAS)}

É um instrumento usado internacionalmente, foi proposto por Watson, Clark e Tellegen (1988) e adaptado para o Brasil por Giacomoni e Hutz (1997), trabalho apresentado em um evento e posteriormente publicado em Zanon e Hutz (2014). É uma escala composta por 20 itens, sendo 10 que avaliam 
afetos negativos e 10 que avaliam afetos positivos, compondo os dois fatores ortogonais da escala: afeto positivo $(\alpha=0,88)$ e afeto negativo $(\alpha=0,86)$. Os itens são constituídos por adjetivos que devem ser respondidos em uma escala de resposta tipo Likert de cinco pontos, sendo 1 "nem um pouco" e 5 "extremamente", em que as pessoas assinalam um número correspondente ao quanto sentem suas emoções descritas pelos adjetivos.

\subsubsection{Satisfaction With Life Scale (SWLS)}

Esta escala foi proposta por Diener, Emmons, Larsen, e Griffin (1985), e adaptada e validada para adultos e adolescentes brasileiros (Giacomoni \& Hutz, 1997). Uma análise fatorial indicou a unidimensionalidade da escala, que é composta por cinco itens que avaliam, de forma global, os aspectos cognitivos do bem-estar subjetivo. Exemplos de itens são: “A minha vida está próxima do meu ideal" e "Até agora eu tenho conseguido as coisas importantes que eu quero na vida". Essa escala apresenta consistência interna adequada $(\alpha=0,91)$. A chave de respostas é uma escala Likert de sete pontos, sendo 1 "discordo plenamente" e 7 "concordo plenamente".

\subsubsection{Escala de satisfação global com relacionamento amoroso}

Escala construída por Rusbult (1983), traduzida e validada por Wachelke, de Andrade, Souza e Moraes (2007) com alto coeficiente alfa de Cronbach $(0,90)$. É composta por três itens respondidos através de uma escala Likert de 5 pontos, sendo 1 = "discordo fortemente" e 5 = "concordo fortemente", sendo eles: "Estou satisfeito com meu relacionamento", "Estou satisfeito com meu(minha) companheiro(a) no que diz respeito a seu papel no relacionamento" e "Estou satisfeito com meu relacionamento com meu(minha) companheiro(a)".

\subsubsection{Questionário Sócio demográfico}

Os participantes responderam a uma série de perguntas de cunho sócio demográfico, a exemplo: sexo, idade, estado civil, religião e estado civil. Este questionário tem por fim caracterizar a amostra.

\subsection{Procedimentos}

Após aprovação pelo comité de Ética competente, foi entregue aos participantes um Termo de Consentimento Livre e Esclarecido - TCLE que foi lido e assinado em duas vias por cada um antes de responderem ao questionário. Ainda se enfatizou o caráter confidencial e sigiloso da participação na pesquisa, assegurando que os resultados só serão apresentados em eventos ou revistas científicas. Um único pesquisador aplicou os questionários, infor- 
mando sobre os objetivos da pesquisa e fornecendo as instruções para o correto preenchimento dos instrumentos. Enfatiza-se que esta pesquisa respeito o presente na declaración de Helsinki, assim como as normas éticas da American Psychological Association (APA).

\subsection{Análise de Dados}

O programa SPSS (versão 22) foi utilizado para realizará de correlação $r$ de Pearson, Regressão Linear e estadísticas descriptivas (medidas de tendencia central, disperso e distribuição de frequências) a fim de caracterizar a amostra.

\section{RESULTADOS}

\subsection{Análises Descritivas}

Foi perguntado aos participantes acerca da sua frequência de uso do smartphone e a frequência com que se sentiam inquietos diante da possibilidade de não poder usar o aparelho. Dentre os participantes, a maioria assumiu usar o smartphone sempre (61,3 \%) seguido de frequentemente $(30,9 \%)$, às vezes $(6,9 \%)$ e raramente $(0,9 \%)$, nenhum participante respondeu que nunca usava. Acerca da inquietação diante da impossibilidade de usar o smartphone, a resposta mais frequente foi "às vezes" $(30,4 \%)$, logo depois veio "frequentemente" (24\%), "sempre" e "raramente" (18\% cada) e "raramente" (9,7\%).

\subsection{Correlações e Regressões}

A primeira análise realizada foi uma correlação $r$ de Pearson entre o partner phubbing, satisfação com o relacionamento e os elementos que compõe o bem-estar subjetivo, ou seja: satisfação com a vida, afetos positivos e afetos negativos. O resultado apresentou correlações significativas entre estas medidas, as quais serão apresentadas na Tabela 1.

Tabela 1.

Correlação entre Partner Phubbing, Afetos Positivos, Afetos Negativos e a Satisfação com a Vida

\begin{tabular}{lccc}
\hline & $\mathbf{1}$ & $\mathbf{2}$ & $\mathbf{3}$ \\
\hline 1. Partner Phubbing & & & \\
2. Afetos Positivos & $-0,32^{* *}$ & & \\
3. Afetos Negativos & $0,33^{* *}$ & $-0,51^{* *}$ & \\
4. Satisfação com a Vida & $-0,13^{*}$ & $0,46^{* *}$ & $-0,38^{* *}$ \\
5. Satisfação com o Relacionamento & $-0,38^{* *}$ & $0,54^{* *}$ & $-0,49^{* *}$ \\
\hline
\end{tabular}

Nota: ${ }^{*} p<0,05, * * p<0,01$ (teste uni-caudal)

Pode-se observar uma correlação negativa e significativa entre partner phubbing e afetos positivos $(r=-0,32, p<0,01)$, satisfação com a vida 
( $r=-0,13 ; p<0,01)$ e satisfação com o relacionamento $(r=-0,38 p<0,01)$, como também apresentou uma correlação significativa, positiva e moderada entre partner phubbing e afetos negativos $(r=0,33 ; p<0,01)$. Este resultado aponta que quando mais o sujeito está exposto ao phubbing por parte do seu parceiro, mais ele experimenta afetos negativos e experimenta menos satisfação com a vida, satisfação com o relacionamento e afetos positivos, ou seja, apresenta menos bem-estar subjetivo e menos satisfação com o seu relacionamento. Apesar de não ser o objetivo do trabalho, torna-se interesante destacar que a análise também apresentou correlações significativas entre afetos positivos e afetos negativos $(r=-0,51 ; p<0,01)$, satisfação com a vida e afetos positivos $(r=0,41 ; p<0,01)$, satisfação com a vida e afetos negativos $(r=-0,38 ; p<0,01)$, satisfação com o relacionamento e satisfação com a vida ( $r=0,41 ; p<0,01)$, satisfação com o relacionamento e afetos positivos ( $r$ $=0,54 ; p<0,01)$ e satisfação com o relacionamento e afetos negativos ( $r=$ $-0,49 ; p<0,01)$. O que sugere que na presença de maior satisfação com o relacionamento, há uma tendencia em se ter maior satisfação com a vida, mais afetos positivos e menos afetos negativos, ao passo que na presença de afetos positivos há uma diminuição nos afetos negativos, o que também acontece com maiores pontuações em satisfação com a vida (diminuição de afetos negativos e aumento nos afetos positivos).

Para aprofundar o conhecimento sobre a influência do partner phubbing sobre a satisfação com o relacionamento, satisfação com a vida, afetos negativos e afetos positivos foram feitas regressões lineares com o partner phubbing como variável independente e a satisfação com o relacionamento, satisfação com a vida, afetos positivos e afetos negativos como variáveis dependentes. Os resultados são apresentados na Tabela 2.

Tabela 2

Regressão Linear

\begin{tabular}{ccccc}
\hline PARTNER PHUBBING & BETA & $\mathbf{R}^{\mathbf{2}}$ & $\mathbf{R}^{\mathbf{2}}$ AJUSTADO & $\mathbf{R}^{\mathbf{2}}$ MUDANÇA \\
\hline Satisfação com o Relacionamento & $-0,38^{*}$ & $0,14^{*}$ & $0,14^{*}$ & $0,14^{*}$ \\
Satisfação com a Vida & $-0,13^{* *}$ & $0,01^{* *}$ & $0,01^{* *}$ & $0,01^{* *}$ \\
Afetos Positivos & $-0,32$ & $0,10^{*}$ & $0,09^{*}$ & $0,10^{*}$ \\
Afetos Negativos & 0,33 & $0,11^{*}$ & $0,11^{*}$ & $0,11^{*}$ \\
\hline
\end{tabular}

Nota: ${ }^{*} p<0,001 ;{ }^{* *} p<0,05$

De acordo com os resultados encontrados, pode-se inferir que a pontuação no partner phubbing explica inversamente a satisfação com o relacionamento ( $\beta=-0,38, t=-6,1, p<0,001)$ e a presença de afetos positivos $(\beta=-0,32 ; t=$ $-4,9 ; p<0,001)$ e explica a presença de afetos negativos $(\beta=0,33 ; t=5,2 ; p<$ 
$0,001)$. No que diz respeito à satisfação com a vida, a pesar de ter significancia estatística, os resultados são muito marginais $(\beta=0,13 ; t=-2,0 ; p<0,05)$. 0 partner phubbing contribuiu para explicar $14 \%$ de variância da satisfação com o relacionamento e cerca de $10 \%$ da variância da presença de afetos positivos de forma direta e negativa, assim como explicou 11 \% da variância dos afetos negativos de forma direta e positiva. De acordo com estes dados, é evidente a influência que o partner phubbing tem para a diminuição na satisfação com o relacionamento e afetos positivos e no aumento dos afetos negativos.

\section{DISCUSIONES}

Este estudo teve como objetivo verificar as relações entre o partner phubbing, o bem-estar subjetivo e a satisfação com o relacionamento. Para isto, utilizando uma amostra de pessoas em algum tipo de relacionamento amoroso, foram realizadas correlação entre partner phubbing, satisfação com a vida e afetos (bem-estar subjetivo), logo após correlação entre partner phubbing e satisfação com o relacionamento o que foi aprofundado com uma regressão linear. A partir do alcançado nos resultados, pode-se afirmar que os objetivos desejados foram alcançados.

Entre os participantes, maioria utilizadores do Smartphone, observou-se que na medida que o phubbing é praticado, as pessoas tendem a experimentar mais afetos negativos e menos positivos e menos satisfação com a vida, como também apresentam menor satisfação no relacionamento. Diante disso, se pode sugerir que a prática do phubbing contribuiu para que as pessoas não façam uma boa avaliação de seu relacionamento amoroso. Estes resultados corroboram com a maioria das pesquisas envolvendo o impacto da tecnologia nos relacionamentos, especialmente as que utilizaram a Partner Phubbing Scale, à começar pela pesquisa realizada pelos próprios autores da escala que após construí-la e validá-la, encontraram correlações negativas entre Pphubbing e a satisfação com a vida, como também negativa entre Pphubbing e satisfação com o relacionamento (Roberts \& David, 2016).

Resultados semelhantes também foram encontrados para a satisfação com o relacionamento. Na China, Wang et al. (2017) encontrou o phubbing como importante fator para a redução da satisfação e consequente depressão; em Portugal, Água et al. (2018) sugere que o phubbing pode reducir a satisfação tanto pelo phubbing em si quanto pelos conflitos resultantes deste comportamento; e em Porto Rico, González-Riviera, Segura-Abreu e UrbistondoRodríguez (2018), apontam que os participantes que apresentaram maior pontuação em phubbing demonstraram menor satisfação em relação ao 
parceiro, menor bem-estar subjetivo, mais sintomas depressivos, estresse e ansiedade.

Assim, pode-se afirmar que, para a amostra estudada, o comportamento de phubbing enquanto se está com o parceiro tem um impacto negativo para as relações, no nível de bem-estar subjetivo e satisfação com o relacionamento, trazendo consequências para a vida a dois e individual de cada parceiro. Segundo a literatura, bem-estar subjetivo e satisfação com o relacionamento estão ligados, uma vez que uma boa avaliação da satisfação com o relacionamento está ligada à maior satisfação com a vida e menos afetos negativos (Snyder, \& Lopez, 2009). Estes resultados podem ser explicados a partir do momento em que as interações entre os parceiros são tidas como um dos mais importantes preditores da satisfação com o relacionamento (Bradbury, Fincham, \& Beach, 2000), assim a necessidade emergente de estar disponível ao smartphone pode parecer ao parceiro que o aparelho merece mais atenção do que ele, gerando conflitos no relacionamento e diminuição na intimidade (McDaniel \& Coyne, 2016; Halpern, \& Katz, 2017). Não é apenas necessário que se esteja na presença do outro, mas que haja conexão entre ambos. Também é importante destacar que estando os parceiros menos satisfeitos com os relacionamentos, estes tendem a ter menos expressão de afetos positivos, menos demonstrações de amor e carinho, assim como menos atividade sexual; o que pode levar ao fim do relacionamento (Gottman, 1998).

Outra forma de se explicar o comportamento de phubbing é tendo em conta a forma cíclica das relações entre as variáveis estudadas. O presente estudo sugere a redução do bem-estar subjetivo decorrente do phubbing sofrido, como também outros estudos apontam o baixo bem-estar subjetivo como um dos fatores determinantes do comportamento de phubbing (Benvenuti, Błachnio, Przepiorka, Daskalova \& Mazzoni, 2019). Ou seja, casais que já se encontram insatisfeitos com sua relação podem se utilizar do smartphone como forma de escape, assim como também o phubbing tem sido apontado como um escape ao tédio (Oduor et al., 2016), solidão, ansiedade e preocupação (Karadag et al., 2015). Então, tamanho engajamento com a tecnologia apontado nos resultados descritivos podem ser motivados por uma necessidade de manejar sentimentos negativos (Kardefelt-Winther, 2014).

Destaca-se a existência de mais determinantes do comportamento de phubbing encontrado na literatura vigente. $\mathrm{O}$ traço de personalidade neuroticismo tem por característica a evitação de contato face a face, o que pode levar os indivíduos a preferir conversas mediadas pela internet e aparelhos 
como o smartphone, tornando o traço de neuroticismo um fator de risco para o phubbing (Kayiş et al., 2016), como mostra o estudo de Erzen, Odaci e Yeniçeri (2019) que aponta uma correlação positiva entre o neuroticismo e o phubbing. Além disto, o phubbing tem estado associado como sendo consequência das dependencias digitais. Karadag et al. (2015) sugere que primeiro o individuo se torna dependente das mídias sociais e internet, o que os leva a depender do smartphone e acaba em se tornar phubbing.

Estes resultados satisfizeram os objetivos do trabalho, servindo para fomentar estudos futuros acerca do phubbing em relacionamentos, em especial em solo brasileiro, assim como fornecendo informações para suprir a necessidade de estudos dos determinantes do bem-estar subjetivo (Umaña, 2007). O impacto do estudo vai além da teoria e se estende em sua relevância social diante da possibilidade de servir como base para políticas voltadas ao uso saudável da tecnología, bem como para intervenções clínicas no âmbito da terapia de casais. Entretanto se faz necessário apresentar algumas limitações presentes, a exemplo da quantidade reduzida de participantes e estes terem sido coletados de forma não probabilística e com maioria com ensino superior completo/incompleto, o que pode afetar a generalização dos resultados. Perante isso, sugere-se que os próximos estudos considerem amostras maiores e com maior diversidade, assim como utilizar outros tipos de análises estatística e investigar as relações com outros construtos, a exemplo da intimidade e conflitos relacionados ao uso do smartphone.

Conflito de Interesse: Não houve conflito de interesse por parte de nenhum autor.

Agradecimientos: Agradecemos à Universidade Federal do Piauí e aos participantes do estudo por permitirem que este fosse possível.

Financiamiento: Não houve financiamento

\section{REFERENCIAS}

Abeele, M. M. V., Antheunis, M. L., \& Schouten, A. P. (2016). The effect of mobile messaging during a conversation on impression formation and interaction quality. Computers in Human Behavior, 62, 562-569. https://doi.org/10.1016/j.chb.2016.04.005

Água, J., Coelho, C., Lourenço, M. G., Patrão, I., \& Leal, I. (2018). Partner Phubbing (Pphubbing): Validação Portuguesa. In Actas do 8o Congresso Internacional de Psicologia da Criança e Adolescente (pp.200-201). http://actas.lis.ulusiada.pt/index.php/ cipca/article/view/684

Benvenuti, M., Błachnio, A., Przepiorka, A., Daskalova, V. e Mazzoni, E. (2019). Factors Related to Phone Snubbing Behavior in Emerging Adults: The Phubbing Phenomenon. In M. Desjarlais (Ed.). The psychology and Dynamics Behind Social Media Interactions (pp.164-187). https://doi.org/10.4018/978-1-5225-9412-3.ch007 
Bradbury, T. N., Fincham, F. D., \& Beach, S. R. (2000). Research on the nature and determinants of marital satisfaction: A decade in review. Journal of marriage and family, 62(4), 964-980. https://doi.org/10.1111/j.1741-3737.2000.00964.x

Cachioni, M., Delfino, L., Yassuda, M., Batistoni, S., Melo, R., \& Domingues, M. (2017). Bem-estar subjetivo e psicológico de idosos participantes de uma Universidade aberta à terceira idade. Revista Brasileira de Geriatria e Gerontologia, 20(3), 340-352. http://dx.doi.org/10.1590/1981-22562017020.160179

Chotpitayasunondh, V., \& Douglas, K. M. (2016). How "phubbing" becomes the norm: The antecedents and consequences of snubbing via smartphone. Computers in Human Behavior, 63, 9-18. https://doi.org/10.1016/j.chb.2016.05.018

Cizmeci, E. (2017). Disconnected, Though Satisfied: Pphubbing Behavior And Relationship Satisfaction. The Turkish Online Journal of Design, Art and Communication, 7(2), 364-375. https://doi.org/10.7456/10702100/018

Deusen, A. J. A. M., Bolle, C. L., Hegner, S. M., \& Kommers, P. A. M. (2015). Modeling habitual and addictive smartphone behavior. The role of smartphone usage types, emotional intelligence, social stress, self-regulation, age, and gender. Computers in human behavior, 45, 411-420. https://doi.org/10.1016/j.chb.2014.12.039

Diener, E., Emmons, R. A., Larsen, R. J. e Griffin, S. (1985). The Satisfaction With Life Scale. Journal of Personality Assessment, 49, 71-5. Retrieved from: https://doi.org/10.1207/ s15327752jpa4901_13

Diener, E., Suh, E. \& Oishi, S. (1998). Recent findings on Subjective Well-Being. Indian Journal of Clinical Psychology, 2, 25-41. http://psycnet.apa.org/record/199743193-002

Erzen, E., Odaci, H., \& Yeniçeri, i. (2019). Phubbing: Which Personality Traits Are Prone to Phubbing?. Social Science Computer Review, 1-14. https://doi. org/10.1177/0894439319847415

Féres-Carneiro, T., \& Ziviani, C. (2009). Conjugalidades contemporâneas: um estudo sobre os múltiplos arranjos amorosos da atualidade. Casal e família: permanências e rupturas. São Paulo: Casa do Psicólogo, 83-107. http://www.puc-rio.br/pibic/ relatorio_resumo2010/resumos/ctch/psi/PSI-Vanessa-Polyana.pdf

Figueiredo, L. B. D. (2016). Tinderelas: busca amorosa por meio de aplicativos para smartphones. https://tede2.pucsp.br/bitstream/handle/18981/2/L\%C3\%ADgia\%20 Baruch\%20de\%20Figueiredo.pdf

Giacomoni, C. H. \& Hutz, C. S. (1997). A mensuração do bem-estar subjetivo: escala de afeto positivo e negativo e escala de satisfação de vida [Resumos]. Em Sociedade Interamericana de Psicologia (Org.). Anais XXVI Congresso Interamericano de Psicologia (p.313). São Paulo: SIP.

Gillmor, C. S. (2007). Stanford, IBM 650 and the first trials of computer date matching. IEEE annals of the history of computing, 29, 74-80. http://doi.org.10.1109/MAHC.2007.13

González-Rivera, J., Segura-Abreu, L., \& Urbistondo-Rodríguez, V. (2018). Phubbing en las Relaciones Románticas: Uso del Celular, Satisfacción en la Pareja, Bienestar Psicológico y Salud Mental. Interacciones, 4(2), 81-91. https://doi.org/10.24016/2018.v4n2.117

Gottman, J. (1998). Casamento: por que alguns dão certo e outros não? São Paulo: Objetiva.

Halpern, D., \& Katz, J. E. (2017). Texting's consequences for romantic relationships: A cross-lagged analysis highlights its risks. Computers in Human Behavior, 71, 386-394. https://doi.org/10.1016/j.chb.2017.01.051

Harrison, M. A., Bealing, C. E., \& Salley, J. M. (2015). 2 TXT or not 2 TXT: College students' reports of when text messaging is social breach. The Social Science Journal, 52(2), 188-194. http://dx.doi.org/10.1016/j.soscij.2015.02.005 
Karadag, E., Tosuntas, S. B., Erzen, E., Duru, P., Bostan, N., Sahin, B. M., Culha, I., Babadag, B. (2015). Determinants of phubbing, which is the sum of many virtual addictions: $A$ structural equation model. Journal of Behavioral Addictions, 4(2), 90-74. https://doi. org/10.1556/2006.4.2015.005

Kardefelt-Winther, D. (2014). A conceptual and methodological critique of internet addiction research: Towards a model of compensatory internet use. Computers in Human Behavior (31:1), pp.351-354. https://doi.org/10.1016/j.chb.2013.10.059

Kayiş, A., Satici, S., Yilmaz, M., Şimşek, D., Ceyhan, E., \& Bakioglu, F. (2016). Big five-personality trait and internet addiction: A meta-analytic review. Computers in Human Behavior, 63, 35-40. https://doi.org/10.1016/j.chb.2016.05.012

Kim, Y., Jeong, J., Cho, H., Jung, D., Kwak, M. Rho, M. J., Yu, H., Kim, D. e Choi, Y. (2016). Personality factors predicting smartphone addiction predisposition: Behavioral inhibition and activation systems, impulsivity, and self-control. Plos One, 11(8). https://doi.org/10.1371/journal.pone.0159788

Lenhart, A., \& Duggan, M. (2014). Couples, the internet, and social media. Pew Internet and American Life Project. https://www.pewinternet.org/2014/02/11/couples-theinternet-and-social-media/

Lucas, R. E., \& Diener, E. (2015). Personality and subjective well-being: Current issues and controversies. In M. Mikulincer, P. R. Shaver, M. L. Cooper, \& R. J. Larsen (Eds.), APA Handbook of Personality and Social Psychology: Volume 4. Personality processes and individual diferences (pp.577-599). Washington, DC, USA: American Psychological Association. https://psycnet.apa.org/record/2013-35883-029

McDaniel, B. T., \& Coyne, S. M. (2016). "Technoference": The interference of technology in couple relationships and implications for women's personal and relational well-being. Psychology of Popular Media Culture, 5(1), 85-98. http://dx.doi.org/10.1037/ ppm0000065

Mcquarie (2017). Mcquarie Dictionary: Australia's national dictionary. Sydney, Australia: Mcquarie Dictionary Publishers.

Meirelles, F. D. S. (2019). 30으 Pesquisa Anual do Uso de TI. https://eaesp.fgv.br/sites/ eaesp.fgv.br/files/pesti2019fgvciappt_2019.pdf

Misra, S., Cheng, L., Genevie, J., \& Yuan, M. (2016). The iPhone effect: the quality of in-person social interactions in the presence of mobile devices. Environment and Behavior, 48(2), 275-298. http://doi.org/10.1177/0013916514539755

Oduor, E., Neustaedter, C., Odom, W., Tang, A., Moallem, N., Tory, M., \& Irani, P. (2016). "The frustrations and benefits of mobile device usage in the home when co-present with family members," in: Proceedings of the 2016 ACM Conference on Designing Interactive Systems-DIS, pp.1315-1327. http://willodom.com/publications/DIS2016/ p1315-oduor_DIS2016.pdf

Ofcom. (2015). The communications market report 2015. England. https://www.ofcom. org.uk/research-and-data/multi-sector-research/cmr/cmr15

Patrão, I. \& Sampaio, D. (2016). Dependências Online: O Poder das tecnologias. Lisboa: Pactor. https://issuu.com/lidel/docs/9789896930608_depend_ncias_online

Perasso, V. (2016). O que é a 4a revolução industrial - e como ela deve afetar nossas vidas. BBC. http://www.bbc.com/portuguese/geral-37658309

Portella, M. R., de Moura Scortegagna, H., Pichler, N. A., \& Graeff, D. B. (2017). Felicidade e satisfação com a vida: voz de mulheres adultas e idosas. Revista Brasileira de Ciências do Envelhecimento Humano, 14(1). https://doi.org/10.5335/rbceh.v14i1.5960

Proulx, C. M., Helms, C. M., \& Buehler, C. (2007). Marital Quality and Personal WellBeing: A Meta-Analysis. Journal of Marriage and Family, 69, 576-593. https://doi. org/10.1111/j.1741-3737.2007.00393.x 
Przybylski, A. K., \& Weinstein, N. (2013). Can you connect with me now? How the presence of mobile communication technology influences face-to-face conversation quality. Journal of Social and Personal Relationships, 30, 237-246. https://doi. org/10.1177/0265407512453827

Rainie, L., \& Zickuhr, K. (2015). Americans' views on mobile etiquette. Pew Research Center, 26. https://www.pewinternet.org/2015/08/26/americans-views-on-mobile-etiquette/

Roberts, J. \& David, M. (2016). My life becomes a major distraction from my cell phone: Partner phubbing and relationship satisfaction among romantic partners. Computers in Human Behavior, 54, 134-141. https:/doi.org/10.1016/j.chb.2015.07.058

Rusbult, C. E. (1983). A longitudinal test of the investment model: The development (and deterioration) of satisfaction and commitment in heterosexual involvements. Journal of Personality and Social Psychology, 45, 101-117. https://doi.org/10.1037//00223514.45.1.101

Shaughnessy, J. J., Zechmeister, E. B., \& Zechmeister, J. S. (2012). Metodologia de pesquisa em psicologia. Brasilia, Brasil: AMGH Editora.

Snyder, C. R., \& Lopez, S. J. (2009). Psicologia positiva: uma abordagem científica e prática das qualidades humanas. (R. C. Costa, Trad). São Paulo: Artmed.

Teixeira, I. N., \& Freire, S. E. A. (in press). Partner Phubbing Scale: Evidências de Validade e Precisão.

Turkle, S.(2011). Alone together: Why we expect more from technology and less from each other. New York:Basic Books. http://alonetogetherbook.com/

Ugur, N \& Koc, T. (2015). Time for Digital Detox: Misuse of Mobile Techonology and phubbing. Social and Behavioral Sciences, 195, 1022-1031. https://foi.org/10.1016/j. sbspro.2015.06.491

Umaña, C. M. (2007). Perspectiva psicológica del bienestar subjetivo. Psicogente, 10(18). http://revistas.unisimon.edu.co/index.php/psicogente/article/view/1559

Viacana, J. J. C., Francisquetti, J. Q., Lima, L. R. D. S., \& de Oliveira Junior, E. (2016). Preciso mexer no celular: a influência do autocontrole e da depleção do ego no uso de smartphones. Revista Brasileira de Marketing, 15(1), 113-132. https://doi.org/ 10.5585/remark.v15i1.2881

Wachelke, J. F., de Andrade, A. L., Souza, A. M. \& Moraes, R. (2007). Estudo complementar da validade fatorial da escala fatorial de satisfação em relacionamento e predição de satisfação global com a relação. PsicoUSF, 12(2), 221-225. http://dx.doi.org/10.1590/ S1413-82712007000200010

Wang, X., Xie, X., Wang, Y., Wang, P., \& Lei, L. (2017). Partner phubbing and depression among married Chinese adults: The roles of relationship satisfaction and relationship length. Personality and Individual Differences, 110, 12-17. https://doi.org/10.1016/j. paid.2017.01.014

Watson, D., Clark, L. A. \& Tellegen, A. (1988) Development and Validation of Brief Measures of Positive and Negative Affect: The PANAS Scales. Journal of Personality and Social Psychology, 69, 719-727. https://doi.org/10.1037/0022-3514.54.6.1063

Zanon, C. \& Hutz, C. S. (2014). Escala de afetos positivos e negativos (Panas). In Hutz, C. S. Avaliação em Psicologia Positiva. Porto Alegre: Artmed.

Esta obra está bajo: Creative commons attribution 4.0 international license. El beneficiario de la licencia tiene el derecho de copiar, distribuir, exhibir y representar la obra y hacer obras derivadas siempre y cuando reconozca y cite la obra de la forma especificada por el autor o el licenciante.

\section{(cc) BY}

